INPLASY

PROTOCOL

To cite: Li et al. Comparison of efficacy and safety of three novel hypoglycemic agents in patients with severe diabetic kidney disease. Inplasy protocol 2021120106. doi: 10.37766/inplasy2021.12.0106

Received: 23 December 2021

Published: 23 December 2021

Corresponding author:

Yiming Mu

muyiming@301hospital.com.cn

Author Affiliation:

Department of Endocrinology, The First Medical Center of PLA General Hospital.

Support: None.

Review Stage at time of this submission: Completed but not published.

\section{Comparison of efficacy and safety of three novel hypoglycemic agents in patients with severe diabetic kidney disease}

Li, Y1; Hu, Y2; Chen, K3; Li, B4; Gu, W5; Mu, Y6.

Review question / Objective: To analyze the efficacy and safety of the three novel hypoglycemics (glucagon-like peptidyl-1 receptor agonists (GLP-1RAs), dipeptidyl peptidase-4 inhibitors (DPP-4i), and sodium-glucose cotransporter 2 inhibitors (SGLT2i)) in type 2 diabetes mellitus(T2DM) patients with severe chronic kidney disease(CKD), based on important RCTs to date.

Condition being studied: The condition being studied was the incidence of severe renal-related AEs or renal death, other outcomes were assessed as serious AEs, hypoglycemia, severe hypoglycemia, all-cause mortality, and changes in HbA1c compared with baseline.

INPLASY registration number: This protocol was registered with the International Platform of Registered Systematic Review and Meta-Analysis Protocols (INPLASY) on 23 December 2021 and was last updated on 23 December 2021 (registration number INPLASY2021120106).

Conflicts of interest:

None declared.

\section{INTRODUCTION}

Review question / Objective: To analyze the efficacy and safety of the three novel hypoglycemics (glucagon-like peptidyl-1 receptor agonists (GLP-1RAs), dipeptidyl peptidase-4 inhibitors (DPP-4i), and sodium-glucose cotransporter 2 inhibitors (SGLT2i)) in type 2 diabetes mellitus(T2DM) patients with severe chronic kidney disease(CKD), based on important RCTs to date.

Rationale: The International Diabetes Federation estimates that $\mathbf{4 6 3}$ million adults aged $200 \mathrm{C} 79$ years had diabetes mellitus (DM) globally in 2019 , and this number is expected to reach 700 million by 2045 . 
Approximately $90 \%$ of patients with DM have type 2 diabetes mellitus (T2DM). Diabetic kidney disease (DKD) accounts for approximately $25 \%$ of diabetic cases and is the leading cause of chronic kidney disease (CKD) and end-stage renal disease (ESRD) in most countries. The pathogenesis of DKD is complex, and its clinical manifestations include a continuous increase in urinary albumin excretion and a progressive decrease in estimated glomerular filtration rate (eGFR). Although there is no cure for CKD, its progress can be delayed by controlling risk factors such as hypertension, hyperglycemia, and dyslipidemia. T2DM in patients with severe CKD (defined in this study as CKD stage 3 B or above, eGFR< $45 \mathrm{~mL} / \mathrm{min} / 1.73 \mathrm{~m} 2$ ) is more challenging to treat since renal damage may affect drug clearance through the kidney, leading to interruption or reduction of most hypoglycemic therapies. In particular, the pharmacological effects of these drugs are difficult to predict in T2DM patients undergoing hemodialysis as the accumulation and rapid clearance of antidiabetic drugs or their metabolites during hemodialysis makes it difficult to maintain blood glucose control. Therefore, it is particularly difficult to provide a scheme for optimal glycemic control in patients with severe CKD. In addition, the control of blood glucose while protecting renal function and reducing mortality is also worthy of further research. In the past few decades, three novel hypoglycemic agents, glucagon-like peptidyl-1 receptor agonists (GLP-1RAs), dipeptidyl peptidase-4 inhibitors (DPP-4i), and sodium-glucose cotransporter 2 inhibitors (SGLT2i) have been introduced. They have become widely used in the treatment of T2DM. The 2020 Guidelines of KDIGO recommended SGLT2i for patients with eGFR "Ż $30 \mathrm{~mL} / \mathrm{min} / 1.73 \mathrm{~m} 2$. GLP-1RAs are safe for patients with CKD but are not recommended for ESRD patients. DPP-4i reduces blood glucose and lowers the risk of hypoglycemia, but there is no evidence that it improves renal or cardiovascular outcomes. After completing several large RCTs, some new findings have been made regarding the efficacy of the three novel hypoglycemics in patients with severe DKD and ESRD. Research by Heerspink et al. revealed that dapagliflozin significantly reduced the incidence of death from renal or cardiovascular causes in DKD patients with eGFR $<45 \mathrm{~mL} / \mathrm{min} / 1.73 \mathrm{~m} 2$ than that in patients who were administered a placebo. Another study showed that dialysis patients taking saxagliptin had significantly lower HbA1c levels than controls. However, there is insufficient evidence to conclude whether these agents are effective for T2DM patients with severe CKD.

Condition being studied: The condition being studied was the incidence of severe renal-related AEs or renal death, other outcomes were assessed as serious AEs, hypoglycemia, severe hypoglycemia, allcause mortality, and changes in HbA1c compared with baseline.

\section{METHODS}

Search strategy: We searched for articles published before July 1, 2021. Electronic searches were conducted in Embase, PubMed/Medline, and the Cochrane Library, The search terms used are : (diabetes OR diabetes mellitus OR

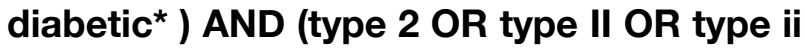
OR NIDDM OR noninsulin dependent OR noninsulin dependent $O R$ adult onset $O R$ mature onset OR late onset ) OR (diabetic nephropath* OR diabetic kidney disease OR renal) AND (glucagon-like peptide 1 receptor inhibitor* OR glucagon-like peptide 1 receptor agonist* OR glucagonlike peptide 1 inhibitor* OR glucagon-like peptide 1 agonist* OR GLP-1 receptor inhibitor ${ }^{\star}$ OR GLP-1 receptor agonist* OR GLP-1 inhibitor* OR GLP-1 agonist* OR dulaglutide OR exenatide OR exendin 4 OR liraglutide OR semaglutide OR taspoglutide OR lixisenatide OR albiglutide OR sodium glucose transporter 2 inhibitor* $O R$ sodium glucose transporter ii inhibitor* OR SGLT 2 inhibitor* OR canagliflozin OR dapagliflozin OR empagliflozin OR ertugliflozin OR tofogliflozin OR bexagliflozin OR henagliflozin OR ipragliflozin licogliflozin OR luseogliflozin OR remogliflozin OR sergliflozin OR sotagliflozin OR dipeptidylpeptidase IV Inhibitor* OR dipeptidyl- 
peptidase 4 Inhibitor* OR DPP4 inhibitor* OR DPP 4 inhibitor* OR DPP IV inhibitor* OR omarigliptin OR vildagliptin OR sitagliptin OR saxagliptin OR linagliptin OR alogliptin OR septagliptin OR gemigliptin OR anagliptin OR teneligliptin OR evogliptin OR dutogliptin OR retagliptin ) AND (cIinicaltrial[Filter] OR randomizedcontrolledtrial[Filter]).

Participant or population: The participants are T2DM patients with severe CKD.

Intervention: Studies included at least one of the following treatments: GLP-1RA (liraglutide only), DPP-4i, andSGLT2i.

Comparator: Positive control drugs or Placebo.

Study designs to be included: RCTs with parallel-group design.

Eligibility criteria: We included RCTs with parallel-group design. The participants were T2DM patients with severe CKD. If the study population in the subgroup met the above criteria, the study was considered eligible. Studies included at least one of the following treatments: GLP-1RA (liraglutide only), DPP-4i, and SGLT2i. The results of eligible studies included changes in HbA1c levels from baseline, hypoglycemic events, renal-related AEs, or death. We excluded single-arm studies and positive control studies for the two drugs in the same categoryWe include RCTs with parallelgroup design. The participants are T2DM patients with severe CKD. If the study population in the subgroup met the above criteria, the study was considered eligible. Studies included at least one of the following treatments: GLP-1RA (liraglutide only), DPP-4i, and SGLT2i. The results of eligible studies included changes in HbA1c levels from baseline, hypoglycemic events, renal-related AEs, or death. We excluded single-arm studies and positive control studies for the two drugs in the same category.

Information sources: We searched for articles published before July 1, 2021. Electronic searches were conducted in
Embase, PubMed/Medline, and the Cochrane Library.

Main outcome(s): The primary outcome was the incidence of severe renal-related AEs or renal death, other outcomes were assessed as serious AEs, hypoglycemia, severe hypoglycemia, all-cause mortality, and changes in HbA1c compared with baseline.

Data management: One author extracted relevant information from qualified trials, and the other author independently reviewed the data. The differences were resolved by consensus through discussion. The extraction included author, year of publication, country, stage of CKD, age, sex percentage, drug use, sample size, and follow-up. For data that were not available in digital form, we used the free software PlotDigitizer to extract digital data from graphics. In some cases, we obtained standard deviations from standard errors or $95 \% \mathrm{Cls}$ and classified data from individual patient data or percentages. We also obtained the required data from publications by different authors of the same trial.

Quality assessment / Risk of bias analysis: The quality of the retrieved RCTs was assessed according to the Cochrane Handbook of Systematic Reviews of Interventions. Potential sources of bias include sequence generation (selection bias), allocation sequence concealment (selection bias), blinding of participants and personnel (performance bias), blinding of outcome assessment (detection bias), incomplete outcome data (attrition bias), and selective outcome reporting (reporting bias). The two authors independently conducted this assessment, and studies were graded as high risk, low risk, or uncertain risk.

Strategy of data synthesis: This network meta-analysis was performed using a frequentist random-effects model. Inconsistency analyses were performed using a design-by-treatment approach. We ranked medications according to their efficacy according to the surface under the 
cumulative ranking (SUCRA). A higher SUCRA value indicated better efficacy. The odds ratio (OR) was used to combine the effect sizes, and interval estimation was performed using $95 \%$ Cls. The level of significance was set at an il value of 0.05 . We evaluated the small-study effects by visually observing publication bias using a comparison-adjusted funnel plot. The mvmeta command and network packages of commands in Stata software were used for this analysis. Pairwise meta-analysis with a random-effects model was used to perform subgroup analysis. All analyses were performed using Stata version 15.0.

Subgroup analysis: We performed a subgroup analysis of patients with ESRD undergoing hemodialysis.

Sensitivity analysis: None.

Language: No language restriction.

Country(ies) involved: China.

Keywords: glucagon-like peptidyl-1 receptor agonists; dipeptidyl peptidase-4 inhibitors; sodium-glucose cotransporter two inhibitors; severe chronic kidney disease; diabetes mellitus.

Contributions of each author:

Author 1 - Yijun Li - conceived and designed the study, performed the statistical analyses.

Author 2 - Ying $\mathrm{Hu}$ - conceived and designed the study, performed the statistical analyses.

Author 3 - Kang Chen - screened and extracted data.

Author 4 - Bing Li - screened and extracted data.

Author 5 - Weijun Gu - conceived and designed the study.

Author 6 - Yiming Mu - conceived and designed the study.

Email: muyiming@301hospital.com.cn 